\title{
Optical Frequency Comb Generation Utilizing Mach-Zehnder Modulator and Multi-Laser Sources
}

\author{
Driss EL Idrissi* and Abdella Battou \\ National Institute of Standards and Technology, USA \\ *Corresponding author: Driss EL Idrissi, National Institute of Standards and Technology, 100 Bureau Dr, Gaithersburg, MD \\ 20899, États-Unis, USA
}

\begin{tabular}{|c|c|}
\hline ARTICLE INFO & ABSTRACT \\
\hline Received: 蔧 July 31, 2019 & stigate a wideband optical frequency comb generator based on multi-laser \\
\hline Published: 蔧 August 09, 2019 & $\begin{array}{l}\text { sources and a single-stage Mach-Zehnder modulator. The generated comb lines were } \\
\text { successfully increased as compared with a single laser. }\end{array}$ \\
\hline
\end{tabular}

Citation: Driss EL Idrissi, Abdella Battou. Optical Frequency Comb Generation Utilizing Mach-Zehnder Modulator and Multi-Laser Sources. Biomed J Sci \& Tech Res 20(3)-2019. BJSTR. MS.ID.003453.

\section{Introduction}

Optical frequency comb generators (OFCG) can offer many attractive applications such as optical communications [1], radio over fiber [1], coherence optical orthogonal frequency-division multiplexing (CO-OFDM) [2], metrology [3] and physics research. The major advantage of the optical frequency comb is the high stability, low noise, low jitter and the fixed frequency spacing which makes it very useful for all these applications. The most popular way of generating a frequency comb is with a mode-locked laser (MLL) [4]. Unfortunately, this conventional approach has a weak stability because of the long-length cavity. This approach is influenced by the environment conditions and the fixed frequency spacing which depends on the cavity length. Other techniques based on semiconductor lasers and high non-linear optical fibers have also been reported [5]. They use photonic crystal fibers for generating new frequencies by four-wave mixing (FWM) and Kerr nonlinearity, using a high optical power to induce non-linear effect in the fibers.

Recently, optical modulation has been used to realize OFCG signals that overcome some of the limitations mentioned above, providing stable and precise optical frequencies with tunable spacing. Optical comb sources based on modulators are good candidates for flexible and stable sources as a result of their operating principle which works without a cavity. A Mach-Zehnder modulator (MZM) approach has been used and demonstrated [6]. The comb spacing, and the bandwidth can be varied by the frequency and the power of an RF signal. The only disadvantage of this technique is mainly due to the limited number of generated comb lines. In order to broadband these comb signals, non-linear optical fibers are typically used. However, this technique requires a high optical power which generates noise and produces nonstability in the comb signal. Recently, other techniques have been proposed and demonstrated using an MZM with an optical feedback loop [7]. In this paper, we propose a new technique to increase the generated comb lines by using multi-laser sources injected simultaneously into an MZM. We also provide a simple equation to define correctly the wavelength of each laser source in order to duplicate the comb signal without a gap. The preliminary results show the multiplication of the generated comb lines as a function of the number of launched laser sources.

\section{Flat optical Frequency Comb Generation}

This section describes the principle of a flattened OFCG based on a single stage MZM. A conventional dual-drive-type MZM fabricated on a $\mathrm{LiNbO}_{3}$ crystal is commonly used to produce multiple sideband on both sides of the center wavelength by optical modulation. As shown in Figure 1a \& 1b, the MZM is driven by two sinusoidal RF signals with a slight difference in the amplitude. An electrical sinusoidal waveform is used to modulate the launched CW laser source in both arms of the MZM. Subsequently, the optical signals, obtained from the two arms, are recombined to generate 
relatively flat comb lines. The spectral spacing and the bandwidth are governed respectively by the RF frequency and the RF power Flat comb signals were obtained when the condition given by the equation below is satisfied [7] (Figure 1).

$$
\Delta A \pm \Delta \theta=\Pi / 2
$$

Where and are respectively, the amplitude difference between the RF signals and the optical phase difference between the two arms of the MZM.

\section{MZ-FCG with Single and Multi-Laser Sources}

In this section we describe the basic configuration of the MZFCG and our proposed system depicted in the Figure 1. The design and the simulation results are obtained using Opti wave commercial software. The first configuration presented in Figure 1a consists of a CW laser and an MZM driven by an RF sinusoidal signal of $10 \mathrm{GHz}$.
In Figure 2a 32 modes were clearly observed with a good flatness, with a spectral spacing of $10 \mathrm{GHz}$. The concept of MZ-FCG, depicted in Figure $2 \mathrm{~b}$ is the duplication of the generated comb lines achieved by a single laser source. Our idea is to generate comb lines from multi-laser sources in the operating wavelength of MZM. For this purpose, multi-laser sources and a WDM multiplexer system is added to the basic configuration. In this case, it is necessary to define correctly the center frequency of each source in order to duplicate the comb lines without gap. We define the center frequency of two successive laser sources by the Equation bellow (Figure 2).

$$
f_{c f}(k)=f_{c f}(k-1)+2 n \Delta f
$$

Where $f_{c f}(k)$ and $f_{c f}(k-1)$ are respectively, the center frequency of two successive laser sources, is the number of sources, is an integer and $\Delta$ is the comb tooth spacing.
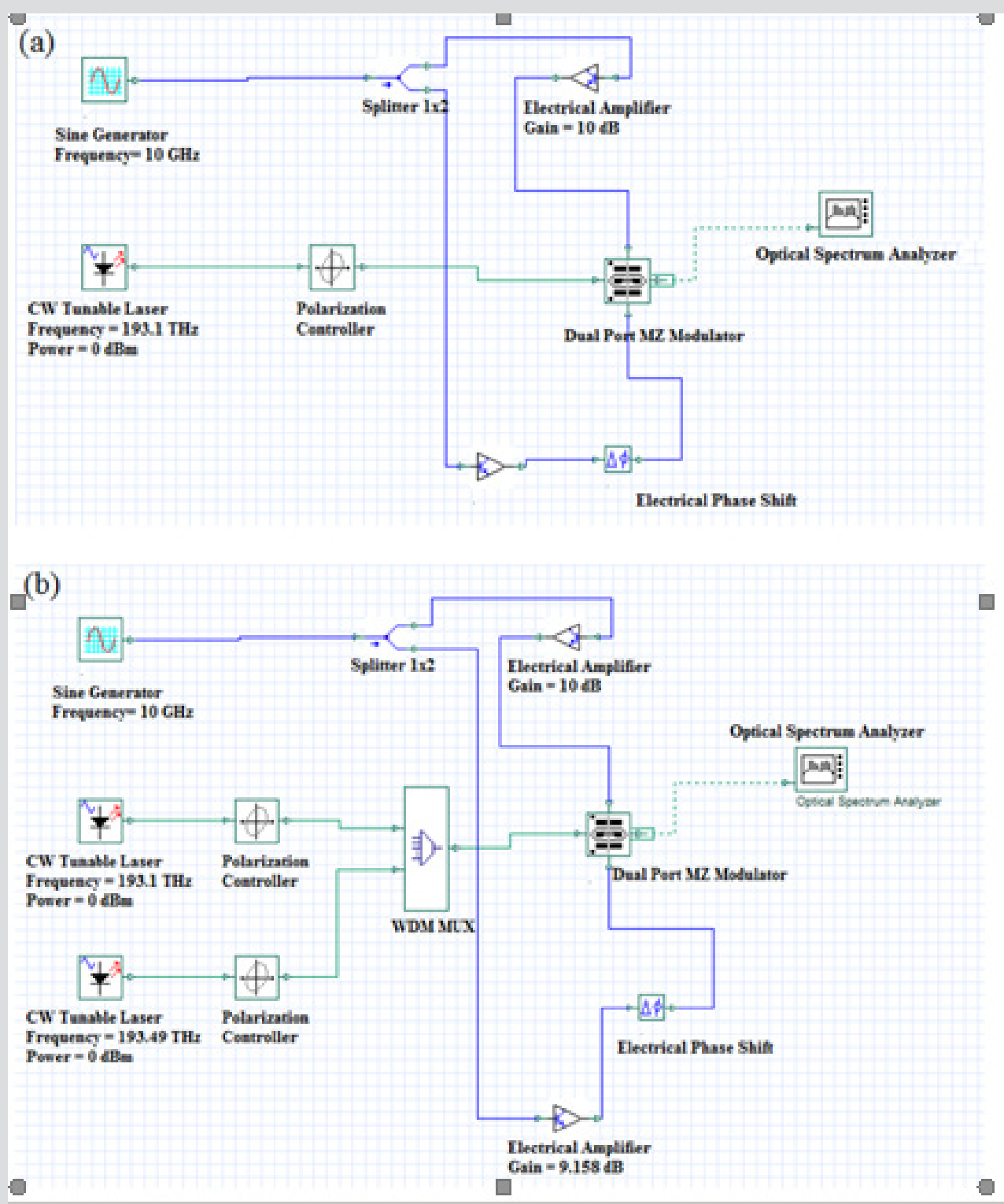

Figure 1: Simulation diagram of OFCG with single and multi-laser sources. 

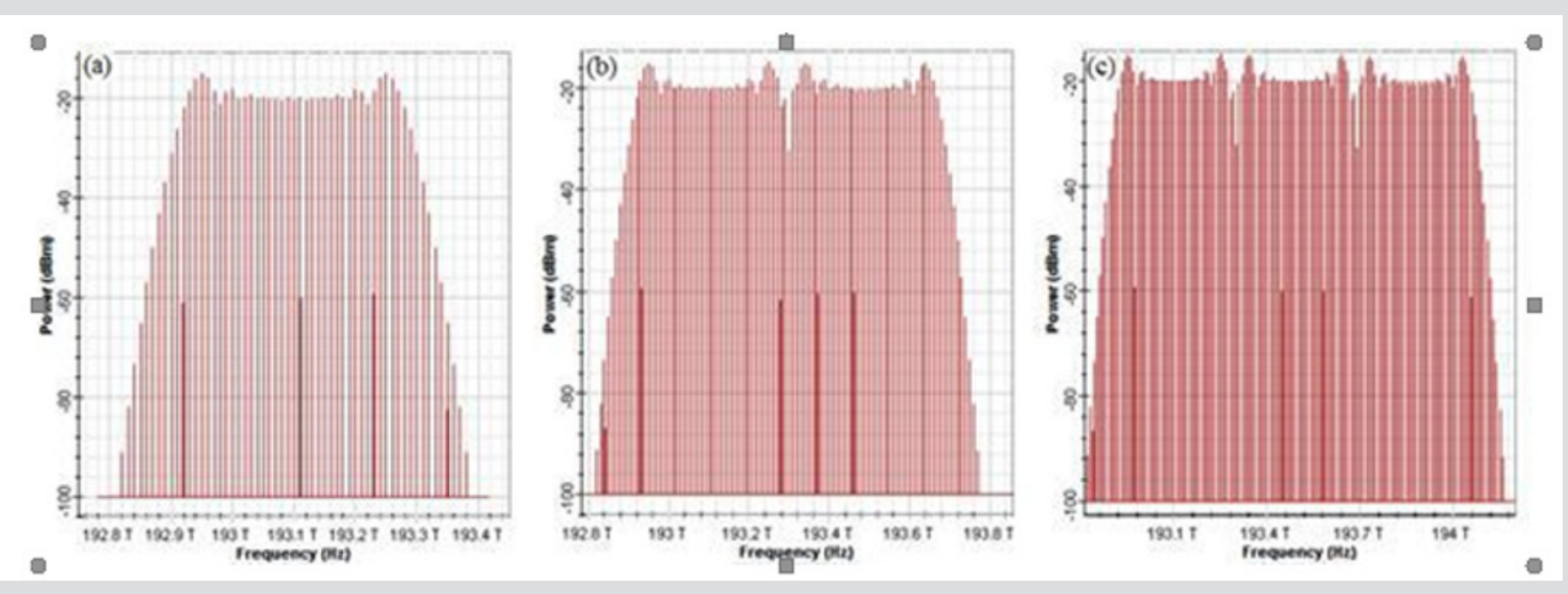

Figure 2: Optical spectra of generated comb signal;

a) with single laser source,

b) with two laser sources and

c) with three laser sources.

Figure $2 \mathrm{~b}$ shows the design of our system with two laser sources. In our simulation the frequency of the first laser source is (1) = 193.1 and according to the Eq. (2) the center frequency of the second laser source is $(2)=193.49$. The simulation result for a single source is shown in Figure $2 \mathrm{a}$, it is clear that 32 comb lines are generated with a good flatness. Figure $2 \mathrm{~b}$ shows the MZ-FCG result based on two multiplexed sources, by using this configuration, the number of comb lines are duplicated. The interference between the different comb teethes which have the same frequency is governed by the phase of the first and second laser sources. In our simulation, we fix the phase of the first laser source and we adjusted the phase of the second laser to obtain a constructive interference in a way to reach a good flatness. Figure $2 \mathrm{c}$ shows the three multiplexed sources result, which multiplied the comb lines by three.

\section{Conclusion}

A new technique for generating a wideband optical frequency comb based on multi-laser sources and a single MZM has been proposed and simulated. By launching simultaneously, more than one laser source using a WDM multiplexing system, the OFCG signal bandwidth was increased.

\section{Acknowledgement}

None.

\section{Conflict of Interest}

No conflict of interest.

\section{References}

1. Chen C, Zhang C, Zhang W, Jin W, Qiu K (2014) Scalable and reconfigurable generation of flat optical comb for WDM-based next-generation broadband optical access networks. Opt. Commun 321: 16-22.

2. Wu C, Zhang X (2006) Impact of Nonlinear Distortion in Radio Over Fiber Systems with Single-Sideband and Tandem Single-Sideband Subcarrier Modulations. J. Lightwave Technol 24(5): 2076-2090.

3. Schibli TR, Minoshima K, Hong FL, Inaba H, Onae A, et al. (2004) Frequency metrology with a turnkey all-fiber system. Opt. Lett 29(21): 2467-2469.

4. Jones DJ, Diddams SA, Ranka JA, Stentz A, Windeler RS, et al. (2000) Carrier-envelope phase control of femtosecond mode-locked lasers and direct optical frequency synthesis. Science 288(5466): 635-639.

5. Morohashi, Sakamoto T, Sekine N, Kawanishi T, Hosako I (2014) Broadband optical comb generation using a MZM-based flat comb generator and a dispersion-shifted fiber for high accurate terahertz wave generation. Engineers Australia 978(1): 21-23.

6. Sakamoto T, Kawanishi T, Izutsu M (2007) Asymptotic formalism for ultraflat optical frequency comb generation using a Mach-Zehnder modulator. Optical Society of America 32(11): 1515-1517.

7. Morohashi I, Sakamoto T, Sotobayashi H, Kawanishi T, Hosako I (2010) Broadband Optical Comb Generation using Mach-Zehnder-ModulatorBased Flat Comb Generator with Feedback Loop. IEEE. 
ISSN: 2574-1241

DOI: 10.26717/BJSTR.2019.20.003453

Driss EL Idrissi. Biomed J Sci \& Tech Res

(C) (P) This work is licensed under Creative

Submission Link: https://biomedres.us/submit-manuscript.php

$\begin{array}{ll}\text { BIOMEDICAL } & \text { Assets of Publishing with us } \\ \text { RESEARCHES } & \text { - Global archiving of articles } \\ & \text { - Immediate, unrestricted online access } \\ & \text { - Rigorous Peer Review Process } \\ \end{array}$

